\title{
The importance of a "socially responsible" approach during COVID-19: the invisible heroes of science in Italy
}

\author{
Filippo Sanfilippo ${ }^{1 *} \mathbb{D}$, Elena Bignami ${ }^{2}$, Ferdinando Luca Lorini ${ }^{3}$ and Marinella Astuto ${ }^{1}$
}

\section{Dear Editor,}

We would like to emphasize the importance of "socially responsible" approaches from physicians and societies during the coronavirus disease-2019 (COVID-19) pandemic. In Italy, a valuable example has been provided by the intensive care (ICU) community. It is important that other disciplines follow such "socially responsible" behavior, as irresponsible communication has generated potentially dangerous consequences. We summarize the "socially responsible" approach of our ICU community in three key points.

Cornerstone has been the avoidance of "public notoriety" at all costs, even when journalists are eager to obtain "shocking" news and notoriety is easily gained. "Shocking" news increase audience, sales, and sharing, but diffusion of unsupported information irresponsibly generates public disorientation with loss of guidance. Ironically, ICU physicians commented we desperately need football games back, so that millions of people become again football managers rather than COVID-19 experts!

The second key point is the identification of few strategic scientists delivering information. Only CrisisUnit Coordinator for Lombardy ICUs and a couple of highly respected scientists who have (or held) apical positions at national and/or international level (SIAARTI and ESICM) were in charge to talk. One of them was recognized as "Healthcare Hero" [1]. Few other experienced physicians (ICU Director in "red areas" of North Italy) fighting COVID-19 on the frontline rarely appeared on TV programs for short communications.

The third key point is the scientific interaction between ICU physicians. They preferred to interact scientifically via webinars with the idea of sharing knowledge gained on the battlefield, generating protocols and, hopefully, improving outcomes of COVID19. Such webinars were responsibly promoted by both SIAARTI and ESICM.

In summary, Italian ICU physicians avoided "compulsory public notoriety," behaving as "invisible heroes of science."

Unfortunately, the same has not happened in other disciplines with compulsory appearance on TV, social media, and newspapers by physicians with low h-index, predatory publication attitude, and no experience in coronavirus delivering highly misleading and scientifically unsupported information. Among other "self-proclaimed experts," we had previous "Nobel-prize candidates" stating the coronavirus will disappear in summer or others reporting $100 \%$ survival on a couple of patients treated with a drug, generating false beliefs in the population. Such approach is "socially irresponsible" and should be stopped.

More than ever, laypeople should be maturely informed. A "socially responsible" approach to public information should be implemented to all fields involved in COVID-19, and the one delivered by the Italian ICU "invisible heroes" should be a leading worldwide example for other disciplines and countries.

\footnotetext{
* Correspondence: filipposanfi@yahoo.it

${ }^{1}$ Department of Anesthesiology and Intensive Care, AOU Policlinico-Vittorio Emanuele, University of Catania, Catania, Italy

Full list of author information is available at the end of the article
} 


\section{Abbreviations}

ESICM: European Society of Intensive Care Medicine; ICU: Intensive care unit; SIAARTI: Società Italiana di Anestesia Analgesia Rianimazione e Terapia Intensiva

\section{Acknowledgements}

We would like to wholeheartedly thank all the healthcare personnel that worked extremely hard under the pressure of this pandemic, always putting patients at the top of their priorities, even before their own health and in some instances the safety of their own families. Unfortunately, we lost a lot of these heroes and they will be sincerely missed.

\section{Authors' contributions}

All authors discussed the content. FS and MA wrote a draft. EB and FLL critically read and suggested changes. All authors read and approved the final version.

\section{Funding}

None

Availability of data and materials

Not applicable

Ethics approval and consent to participate

Not applicable

\section{Consent for publication}

Not applicable

\section{Competing interests}

None

\section{Author details}

${ }^{1}$ Department of Anesthesiology and Intensive Care, AOU Policlinico-Vittorio Emanuele, University of Catania, Catania, Italy. ${ }^{2}$ Anesthesiology, Critical Care and Pain Medicine Division, Department of Medicine and Surgery, University of Parma, Parma, Italy. ${ }^{3}$ Emergency and Intensive Care Department, ASST

Papa Giovanni XXIII, Bergamo, Italy.

Received: 7 May 2020 Accepted: 18 May 2020

Published online: 26 May 2020

\section{Reference}

1. Bauchner H, Easley TJ. Health care heroes of the COVID-19 pandemic. JAMA. 2020. https://pubmed.ncbi.nlm.nih.gov/32310289/?from_single_result= Bauchner+H\%2C+Easley+TJ.+Health+care+heroes+of+the+COVID-19+ pandemic.+JAMA+2020.\&expanded_search_query=Bauchner+H\%2C+ Easley+TJ.+Health+care+heroes+of+the+COVID-19+pandemic.+JAMA+2020.

\section{Publisher's Note}

Springer Nature remains neutral with regard to jurisdictional claims in published maps and institutional affiliations. 